\title{
Early Response to Antipsychotic Drug Therapy as a Clinical Marker of Subsequent Response in the Treatment of Schizophrenia
}

\author{
Bruce J Kinon*,', Lei Chen', Haya Ascher-Svanum', Virginia L Stauffer', Sara Kollack-Walker', \\ Wei Zhou', Shitij Kapur' and John M Kane ${ }^{3}$ \\ 'Lilly Research Laboratories, Eli Lilly and Company, Indianapolis, IN, USA; ${ }^{2}$ Institute of Psychiatry, King's College of London, London, UK; ${ }^{3}$ Zucker \\ Hillside Hospital, Glen Oaks, NY, USA
}

\begin{abstract}
Our objective was to prospectively assess whether early (ie, 2 weeks) response to an antipsychotic predicts later (12-week) response and whether 'switching' early non-responders to another antipsychotic is a better strategy than 'staying'. This randomized, double-blind, flexible-dosed, 12-week study enrolled 628 patients diagnosed with schizophrenia or schizoaffective disorder. All initiated treatment with risperidone. Early response was defined as $\geqslant 20 \%$ improvement on the Positive and Negative Syndrome Scale (PANSS) total score following 2 weeks of treatment. Early responders (ERs) continued on risperidone, whereas early non-responders (ENRs) were randomized $(1: \mid)$ to continue on risperidone $2-6 \mathrm{mg} /$ day or switch to olanzapine $10-20 \mathrm{mg} /$ day for 10 additional weeks. Compared with ENRs, risperidone ERs showed significantly greater reduction in PANSS total score (end point; $p<00 \mathrm{I}$ ). Early response/nonresponse was highly predictive of subsequent clinical outcomes. Switching risperidone ENRs to olanzapine at week 2 resulted in a small but significantly greater reduction in PANSS total score (end point; $p=0.020$ ) and in depressive symptoms (end point; $p=0.004$ ); the reduction in PANSS was greater among those who were still moderately ill at 2 weeks. Switching risperidone ENRs to olanzapine also resulted in significantly greater increases in triglycerides, a significantly greater decrease in prolactin, and significantly less treatmentemergent dyskinesia. This is the first study to prospectively show that early response/non-response to an antipsychotic (risperidone) is a reliable clinical marker of subsequent clinical outcomes and that a 'switching' strategy based on this information may lead to greater clinical improvement than staying on a drug for a longer period in some patients.
\end{abstract}

Neuropsychopharmacology (20 I0) 35, 58I-590; do::I0.1038/npp.2009. I64; published online 4 November 2009

Keywords: schizophrenia; risperidone; early response; atypical antipsychotic; prediction

\section{INTRODUCTION}

One of the most challenging issues in treating psychotic exacerbations in schizophrenia is deciding how long an initial trial of an antipsychotic should last and what response metrics should be used to inform that decision. Although for nearly four decades the field of schizophrenia has viewed antipsychotic drug response as delayed, recent research has showed that the onset of response can occur

\footnotetext{
*Correspondence: Dr BJ Kinon, Lilly Research Laboratories, Eli Lilly and Company, Lilly Corporate Center, DC 6166, Indianapolis, IN, 46285, USA, Tel: + I 317277 7886, Fax: + I 317433 0448, E-mail: Kinon_Bruce@Lilly.com

Previous Presentation: These data debuted at the annual American Psychiatric Association (APA) congress in Washington, DC, May 3-8, 2008.

Clinical Trials Registration: ClinicalTrials.gov identifier: NCT00337662; http://www.clinicaltrials.gov/

Received 7 April 2009; revised 28 August 2009; accepted 15 September 2009
}

rapidly within the first week or two (Agid et al, 2003; Leucht et al, 2005a). Compared with patients who lack at least minimal symptom improvement following 2 weeks of treatment ('early non-responders'), early responders were previously found to have greater improvement in symptoms and functioning, a higher symptom remission rate (AscherSvanum et al, 2008; Kinon et al, 2008) and lower health care costs (Ascher-Svanum et al, 2008). Thus, it is important when possible to identify patients as early responders or early non-responders, and consider alternative treatment options for patients who are less likely to respond.

To date, all of the studies on early response/non-response have been retrospective in nature, and have shown that early non-response is a robust predictor of subsequent nonresponse with continued treatment of the same medication (Ascher-Svanum et al, 2008; Correll et al, 2003; Kinon et al, 2008; Leucht et al, 2007; Leucht et al, 2008). These studies also show that a majority of patients (nearly $70 \%$ ) do not reach this 'early response' criterion with either a typical or atypical antipsychotic drug (Ascher-Svanum et al, 2008; 
Kinon et al, 1993; Kinon et al, 2008). The clinical dilemma in these patients who do not show early response is whether one should 'switch' or 'stay'. Although favorable outcomes were previously reported for patients with a poor or limited response to one antipsychotic drug following a switch to another (Canive et al, 2006; Simpson et al, 2008; Wang et al, 2006; Takahashi et al, 2006a; Takahashi et al, 2006b), such studies typically lack a control group (of patients staying on the original drug for an equal interval) and, therefore, we cannot conclude that switching is indeed a beneficial option. Importantly, no benefit of switching antipsychotic medications was reported in two post hoc analyses of the Clinical Antipsychotic Trials of Intervention Effectiveness (CATIE) trial that included a control group (Essock et al, 2006; Rosenheck et al, 2009). Considering the mixed results and methodological issues, additional research that is prospective and incorporates a randomized, double-blind control group is needed to assess whether switching antipsychotic drugs can be beneficial following failure to achieve symptom improvement early in treatment.

The objectives of this study were to prospectively assess whether early response to one atypical antipsychotic drug is a clinical marker of subsequent symptom improvement, and if switching early non-responders to another atypical antipsychotic would facilitate improvement in overall psychopathology and depressive symptoms. With the recent patent expiration of risperidone in the US, we anticipated that clinicians may consider treatment algorithms, which advise a 'fail-first' approach with risperidone before switching to another antipsychotic drug. Therefore, we chose to study early response/non-response initially in patients treated with risperidone, thus addressing a question of considerable practical relevance. We selected olanzapine as the 'switch to' agent based, in part, on studies reporting greater efficacy or effectiveness of olanzapine treatment compared with risperidone (Alvarez et al, 2006; Dossenbach et al, 2004; Lieberman et al, 2005; Takahashi et al, 2006a; Tran et al, 1997), though it has to be said that data to the contrary also exist (Conley and Mahmoud, 2001; Davis et al, 2003).

In our 12-week study, patients were treated with flexibledose oral risperidone for 2 weeks and were then divided into early responders and early non-responders based on an a priori cutoff of $20 \%$ improvement in PANSS total scores. Early responders to risperidone (ERs) continued with risperidone therapy, whereas early non-responders to risperidone (ENRs) were randomized in a double-blind manner to either continue on risperidone (ENR-RIS) or switch to olanzapine (ENR-OLZ) for the next 10 weeks. In a prospective manner, we tested the following hypotheses: (1) patients who were ERs would show greater improvement in psychopathology compared with ENRs; (2) response/non-response at 2 weeks would predict subsequent clinical outcomes; and (3) patients who were ENRs and then switched to olanzapine would show significantly greater improvement in overall psychopathology and depressive symptoms compared with those staying on risperidone. Questions 1 and 2 would validate the use of the $20 \%$ improvement by 2 weeks criterion as the decision-making threshold, and Question 3 would answer whether switching/staying based on this criterion was clinically useful.

\section{MATERIALS AND METHODS}

\section{Study Design}

This was a randomized, double-blind, flexible-dose, parallel, 12 -week study to explore the relationship between early response to an antipsychotic medication and subsequent improvement in psychopathology using the oral atypical antipsychotic risperidone (Figure 1a). Patients from 18-65 years of age met diagnostic criteria for schizophrenia, schizoaffective disorder, or schizophreniform disorder according to the Diagnostic and Statistical Manual of Mental Disorders, Fourth Edition (DSM-IV). After receiving a

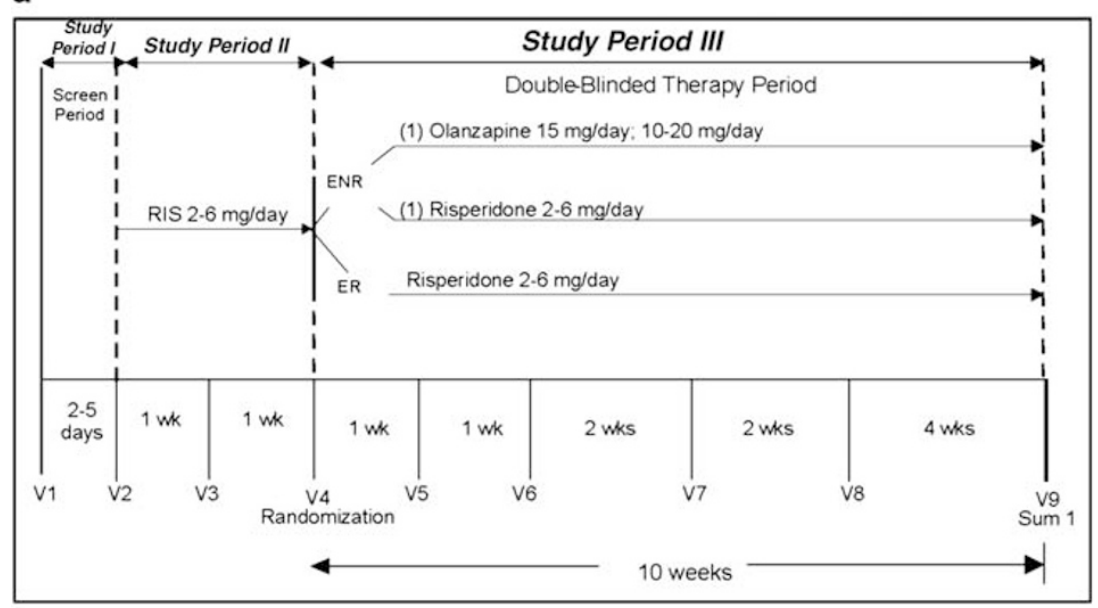

[RIS Early Responders (ER)]

[RIS Early Non-Responders (ENR)] b

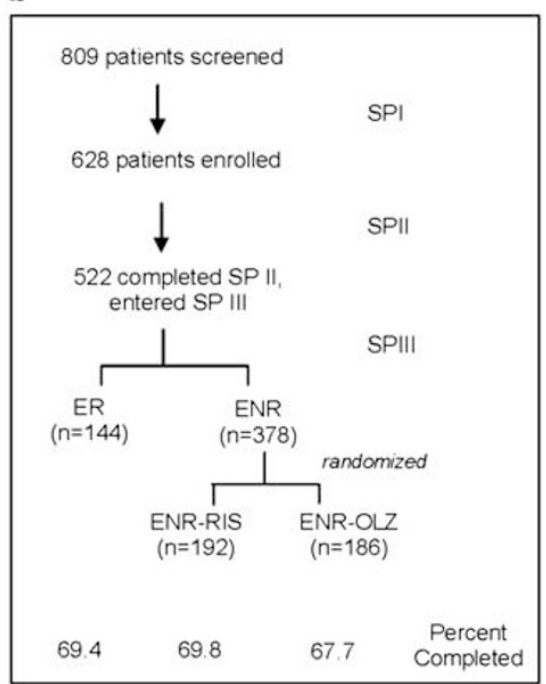

Figure I (a) Study design. (b) Patient flow diagram. ER, early responder; ENR, early non-responder; ENR-OLZ, early non-responder switched to olanzapine; ENR-RIS, early non-responder maintained on risperidone; mg, milligram; SPI, Study Period I; SPII, Study Period II; SPIII, Study Period III; V, Visit; wk, week. 
a complete description of the study, eligible patients provided written informed consent before undergoing any study procedure or receiving any study treatment. In addition, the study was approved by the Ethics Committee from each participating institution and the study was conducted in accordance with the Declaration of Helsinki. This study was conducted at 64 centers in three countries, starting May 2006 and ending December 2007.

During Study Period I, moderately ill patients in whom a switch to another antipsychotic medication was acutely indicated were assessed for study participation through physical, historical, and psychiatric assessments. Patients had to meet the following psychopathology severity criteria at Visit 1: (1) Brief Psychiatric Rating Scale (BPRS) (Overall and Gorham, 1962) total score extracted from the Positive and Negative Syndrome Scale (PANSS) (Kay et al, 1987) of at least 45 (18-item version, in which one indicates 'absent' and seven indicates 'severe'); (2) a score of at least 4 (moderate) on at least two of the following BPRS items: conceptual disorganization, suspiciousness, hallucinatory behavior, and unusual thought content; and (3) a minimum Clinical Global Impressions-Severity (CGI-S) scale (Guy, 1976) rating of 4 (moderately ill) or greater. Patients had to have experienced an exacerbation of their illness within the 2 weeks preceding Visit 1 that required a change in the level of psychiatric care including, but not limited to: inpatient hospitalization, day/partial hospitalization, outpatient crisis management, or psychiatric treatment in an emergency room. Patients were excluded from study participation if they had: a history of an inadequate response to risperidone or olanzapine; an allergic reaction to risperidone or olanzapine; received risperidone or olanzapine or an experimental drug within the past 30 days before study enrollment; been hospitalized for greater than 2 weeks immediately before visit 1 ; or other acute, serious, or unstable medical conditions.

During Study Period II, patients began single-blind (ie, to dose and dose adjustments), flexible-dose therapy at Visit 2 with risperidone $2-6 \mathrm{mg} /$ day taken in the evening. Patients were abruptly switched from their current antipsychotic medications to risperidone titrated as follows: $2 \mathrm{mg} / \mathrm{day}$ on day $1,4 \mathrm{mg} /$ day on days $2-7$, and flexible dosing $2-6 \mathrm{mg} / \mathrm{day}$ onward. At 2 weeks, patients were classified as ERs or ENRs based on the a priori criterion of early response/nonresponse. To minimize information bias, the precise response criterion was withheld from research staff (but defined a priori in the Institutional Review Board (IRB) Supplement, and early responder/non-responder status was identified and treatment randomization was implemented using an interactive voice response (IVR) system).

During Study Period III (weeks 3-12), patients who were ERs continued to receive risperidone for the duration of the study. Patients who were ENRs were randomized $(1: 1)$ to either stay on risperidone (ENR-RIS: $2-6 \mathrm{mg}$ /day) or switch to olanzapine (ENR-OLZ: 10-20 mg/day) for 10 weeks. As the treating physicians did not know the formal responder/ non-responder status, they continued to optimize the patients' outcomes without knowledge of any change in treatment group or drug assignment.

The use of benzodiazepine/hypnotics/anxiolytics was permitted during the study, but only for the treatment of anxiety or insomnia as clinically indicated. Patients receiving a stable dose of antidepressants, anticonvulsants used as a mood stabilizer, or lithium therapy for at least 30 days before study initiation could continue on these concomitant medications at a stable dose. However, the doses of these medications could not be changed in an attempt to enhance efficacy.

\section{Measures}

The primary objective was to determine if early onset of response to risperidone treatment is associated with greater subsequent improvement in psychopathology compared with lack of early onset of response. Patients were assigned into ER or ENR groups at 2 weeks based on a priori-defined improvement in the PANSS $_{1-7}$ total score. ERs showed $\geqslant 20 \%$ improvement in PANSS total score from baseline. ENRs showed $<20 \%$ improvement in PANSS total score from baseline. The primary outcome measure was change in PANSS total score between ERs and ENRs during the subsequent 10-week period (Study Period III; visits 5-9, weeks 3-12).

A valid criterion for prediction has to include two a priori elements, the duration of treatment and the degree of improvement, to use as a threshold. We chose a treatment duration of 2 weeks based on previous studies showing that significantly greater improvement in symptoms occurs during weeks 1 and 2 of treatment compared with weeks 3 and 4 (Agid et al, 2003; Leucht et al, 2005a). The cutoff of $20 \%$ improvement was based on research showing that 'minimal improvement' on the CGI-S scale is associated with $23 \%$ reduction in the PANSS total score at 2 weeks (Leucht et al, 2005b), and is consistent with previous studies assessing the relationship between early response/ non-response and subsequent treatment outcomes (AscherSvanum et al, 2008; Correll et al, 2003; Kinon et al, 2008). The sensitivity, specificity, and predictive accuracy of early response were determined using an a priori definition of final response as $\geqslant 20, \geqslant 30$, and $\geqslant 40 \%$ improvement in PANSS total score (moderate improvement) at 12 weeks.

In this study, we also report on the secondary outcome of switching on overall psychiatric symptoms to determine if the trajectory of a limited response profile for ENRs treated with one antipsychotic drug can be improved when switched to another. This secondary outcome measure assessed the change in PANSS total score between ENR-RIS and ENR-OLZ groups during the subsequent 10-week period (Study Period III; visits 5-9, weeks 3-12). Another secondary outcome measure reported in this paper was change in the Montgomery-Asberg Depression Rating Scale (MADRS) (Montgomery and Asberg, 1979) to measure the severity of depressive symptoms. Additional secondary measures were included in this clinical trial (eg, measures of cognition, subjective well being, and functioning), and will be reported in a separate article.

Safety measurements were taken to assess vital signs, changes in various laboratory measures, and changes in extrapyramidal symptoms. Scales designed to measure extrapyramidal symptoms included the modified version of the Simpson-Angus Scale (Simpson and Angus, 1970) for parkinsonian symptoms, the Barnes-Akathisia Scale 
(Barnes, 1989) for akathisia, and the Abnormal Involuntary Movement Scale (Guy, 1976) for tardive dyskinesia.

\section{Statistical Methodology}

Baseline demographic characteristics, psychiatric history, and disease severity were compared between ERs and ENRs by Fisher's exact test for categorical variables and by analysis of variance (ANOVA) for continuous variables. The rates of early treatment discontinuation were compared between ERs and ENRs by Fisher's exact test.

Mixed-effects Model Repeated Measure (MMRM) analyses were implemented on the change in PANSS total score up to 12 weeks, comparing ER $v s$ ENR groups at each visit during Study Period III. The MMRM model included fixed terms of baseline PANSS total score, early response/nonresponse group, time (visit), time-by-group interaction, and investigator. Changes in the PANSS total score during Study Period II were analyzed by analysis of covariance (ANCOVA) with the terms of treatment group, investigator, and baseline PANSS total score.

Sensitivity, specificity, positive predictive value (PPV), negative predictive value (NPV), and total accuracy of predicting response/non-response at end point by early response status at Week 2 were calculated with response defined as a positive event. Sensitivity is the proportion of ultimate responders who were correctly classified as ERs at 2 weeks. Specificity is the proportion of ultimate non-responders who were correctly classified as ENRs at 2 weeks. PPV is the proportion of ERs who remained 'responders' at end point. NPV is the proportion of ENRs who remained 'non-responders' at end point. Total accuracy is the proportion of patients whose 2-week response status (early response or early non-response) accurately predicted subsequent response status. As half of the ENRs were randomly switched to olanzapine at the end of week 2 , this analysis only included patients who maintained on risperidone treatment with a weighting of $1: 2$ assigned to the ER and ENR groups, respectively.

MADRS was analyzed for Study Period II and III separately using ANCOVA models with the terms of treatment group, investigator, baseline PANSS total score, and baseline MADRS total score.

The percentage of patients in each group who achieved subsequent response at end point was noted and compared using Fisher's exact test, as well as using logistic regression accounting for baseline PANSS total score.

Changes from baseline to end point in laboratory values and weight were analyzed for within-group change with a Wilcoxon Signed Rank test and for between-group differences with a ranked ANOVA model adjusted for investigator. Treatment-emergent extrapyramidal symptoms were analyzed by Fisher's exact test.

For the comparison of ER vs ENR-RIS groups, data collected at Visit 1 or 2 were used as baseline. For the comparison of ENR-RIS $v s$ ENR-OLZ groups, Visit 4 was used as baseline. Unless otherwise specified, missing values were handled by the method of last-observation-carriedforward (LOCF).

\section{RESULTS}

\section{Patient Characteristics}

A total of 628 patients enrolled in the study, of which 522 completed Study Period II and entered Study Period III. The baseline demographic characteristics, psychiatric history, and disease severity of patients in the early responder and early non-responder groups are shown in Table 1. Patients were chronically ill, diagnosed predominately with schizophrenia, with about 18 years of illness duration. Compared with ERs, ENRs had a significantly greater number of previous episodes of schizophrenia in the last 24 months, a significantly lower mean PANSS total score, and a significantly lower PANSS positive subscore. No differences in the number of patients receiving typical or atypical medications or the number receiving no medication was observed between ERs and ENRs at screening (data not shown).

\section{Patient Disposition}

Approximately $17 \%$ of patients (106/628) discontinued risperidone treatment before the end of the first 2 weeks (Study Period II). Reasons for early study discontinuation during these first 2 weeks included: lost to follow up $(n=35,5.6 \%)$, subject decision $(n=20,3.2 \%)$, adverse event $(n=16,2.6 \%)$, physician decision $(n=13,2.1 \%)$, lack of efficacy $(n=6,1.0 \%)$, entry criteria not met $(n=6$, $1.0 \%)$, protocol violation $(n=6,1.0 \%)$, sponsor decision $(n=2,0.3 \%)$, and parent/caregiver decision $(n=1,0.2 \%)$.

Among those patients who completed 2 weeks of treatment, $72.4 \%$ of patients were in the ENR group, whereas $27.6 \%$ were in the ER group. The ENRs had a significantly higher mean modal dose of risperidone during the first 2 weeks (ER group $(n=144): 4.5 \mathrm{mg} /$ day; ENR group $(n=378): 4.8 \mathrm{mg} /$ day; $p=0.002)$. A summary diagram of patient flow through the study is provided (Figure 1b).

For all patients who entered Study Period III, no significant group differences were observed in the rate of early study discontinuation: $30.6 \%$ of patients discontinued early in the ER group, $30.2 \%$ of patients discontinued early in the ENR-RIS group, and $32.3 \%$ of patients discontinued early in the ENR-OLZ group. No significant differences were observed in reasons for early study discontinuation between the ER and ENR-RIS groups, or between the ENRRIS and ENR-OLZ groups (data not shown).

\section{Efficacy Measures}

Consistent with our hypothesis and the primary objective of this study, the ER group showed, from Week 2 to end point, a significantly greater reduction in the PANSS total score at all subsequent time points compared with the ENR-RIS group (Figure 2). Although the MMRM analyses were adjusted for baseline differences, it was not possible to use LS means to construct the figure and corresponding table as the LS means are dependent on the specific comparisons made. Therefore, shown in the figure and table are mean changes in the PANSS total score in each group. The values differ slightly from the LS mean values provided in the text below. 


\begin{tabular}{|c|c|c|c|}
\hline & $\begin{array}{c}\text { Early responders } \\
\text { to Risperidone }(N=\mid 44)\end{array}$ & $\begin{array}{l}\text { Early non-responders } \\
\text { to Risperidone }(N=378)\end{array}$ & $p$-value \\
\hline Age (years), mean (SD) & $41.8(10.9)$ & $41.9(11.1)$ & 0.980 \\
\hline Ethnicity/race: & & & 0.191 \\
\hline Caucasian, \% & 51.4 & 42.3 & - \\
\hline \multicolumn{4}{|l|}{ Diagnosis: } \\
\hline Schizophrenia/schizophreniform, \% & 79.2 & 75.1 & 0.359 \\
\hline Schizoaffective bipolar, \% & 14.6 & 14.8 & 1.000 \\
\hline Schizoaffective depression, \% & 6.3 & 10.1 & 0.231 \\
\hline PANSS Total, mean (SD) & $93.9(13.0)$ & $91.2(\mid 3.8)$ & 0.030 \\
\hline PANSS Positive, mean (SD) & $25.5(4.1)$ & $23.7(3.8)$ & $<0.001$ \\
\hline PANSS Negative, mean (SD) & $22.9(4.4)$ & $22.2(5.2)$ & 0.111 \\
\hline MADRS Total, mean (SD) & $15.9(10.0)$ & I $6.4(8.7)$ & 0.759 \\
\hline Weight $(\mathrm{kg})$, mean & 90.5 & 88.5 & 0.409 \\
\hline
\end{tabular}

MADRS, Montgomery-Asberg Depression Rating Scale; N, number of patients; $n$, number in group; PANSS, Positive and Negative Syndrome Scale.

In addition, the ER group showed significantly greater reductions from baseline to end point in PANSS positive, negative, and general psychopathology subscores compared with the ENR-RIS group $(p<0.001$ ) (data not shown). ERs were also significantly more likely to attain the threshold of normal to mild on the CGI-S scale, whereas patients in the ENR-RIS group were significantly more likely to remain moderately ill or worse at 12 weeks $(p<0.001$, LOCF) (data not shown). Compared with ENR-RIS group, a larger proportion of ERs achieved subsequent therapeutic response defined as $\geqslant 20,30,40$, and $50 \%$ improvement in PANSS total score $(p<0.001)$ at end point (data not shown).

Compared with the ENR-RIS group, ERs achieved a greater reduction in depressive symptoms (baseline to end point mean change in MADRS, $p<0.001$ ) (data not shown).

\section{Prediction of Final Response}

Using an a priori definition of $\geqslant 30 \%$ improvement as criterion for subsequent response at 12 weeks (Table 2), we found that $87.0 \%$ of non-responders at end point were correctly identified as ENRs at 2 weeks (specificity); and $76.2 \%$ of ENRs were non-responders at end point (NPV). Furthermore, $51.4 \%$ of responders at end point were correctly identified as ERs at 2 weeks (sensitivity); and $68.8 \%$ of ERs were responders at end point (PPV). The overall predictive accuracy was $74.2 \%$.

To examine whether the predictive characteristics of our 2-week $20 \%$ cutoff score would change with a different definition of final response, we performed a sensitivity analysis to examine the effect of defining the 12-week response as either at least 20 or $40 \%$ improvement in PANSS total score. The higher end point value led to an even higher sensitivity (63.0\%), but at the expense of lower specificity $(81.1 \%)$, with relatively similar overall predictive value $(77.9 \%)$.

\section{Switching $v s$ Staying}

Switching risperidone early non-responders to olanzapine resulted in a significantly greater reduction in PANSS total score that reached significance at end point (least-squares (LS) mean change at week 12: ENR-RIS, -13.53; ENR-OLZ, $-17.02 ; p=0.020$ ) (Figure 2). A comparison of the proportion of responders observed at end point between the ENR-RIS and ENR-OLZ groups did not reveal significant between-group differences for categorical response criteria of 20,30 , or $40 \%$ reduction in PANSS total score at end point, although a significantly greater proportion of patients who switched to olanzapine attained at least a $50 \%$ reduction in symptoms (Figure 3 ).

In addition, switching risperidone early non-responders to olanzapine resulted in significantly greater reduction in MADRS (ENR-RIS, -1.02; ENR-OLZ, -2.73 ; end point; $p=0.020$ ). The proportion of ENRs with a categorical assessment of moderate to severe depressive symptoms (MADRS > 15) was significantly lower for ENR-OLZ group compared with ENR-RIS group (ENR-RIS, 29.2\%; ENROLZ, 23.2\%; end point; $p=0.033$ ).

\section{Secondary Subgroup Analysis: Early Non-Responders who Remained Moderately Ill at Week 2}

It is possible that a subset of ENRs had just missed the $20 \%$ improvement in PANSS total score after the first 2 weeks of treatment. If so, they may not have been able to show as 


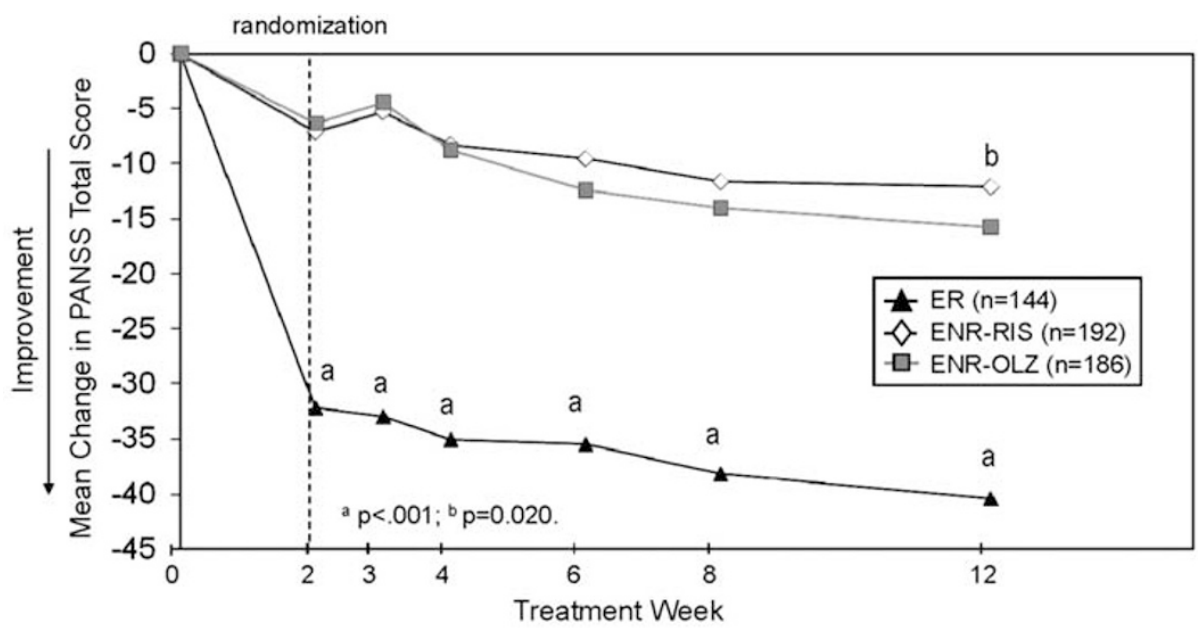

\begin{tabular}{|l|c|c|c|c|c|c|c|}
\hline & \multicolumn{7}{|c|}{ Week } \\
\hline & 0 & 2 & 3 & 4 & 6 & 8 & 12 \\
\hline ER $(\mathrm{n}=144)$ & 0 & -32.22 & -33.01 & -35.08 & -35.49 & -38.13 & -40.39 \\
\hline ENR-RIS $(\mathrm{n}=192)$ & 0 & -7.1 & -5.29 & -8.29 & -9.55 & -11.62 & -12.09 \\
\hline ENR-OLZ $(\mathrm{n}=186)$ & 0 & -6.36 & -4.44 & -8.78 & -12.41 & -14.04 & -15.78 \\
\hline
\end{tabular}

Figure 2 Comparison of change in PANSS total score from baseline between early responders and early non-responders treated with risperidone and between early non-responders maintained on risperidone (ENR-RIS) and those switched to olanzapine (ENR-OLZ). PANSS, Positive and Negative Syndrome Scale; RIS, risperidone.

Table 2 Receiver-Operating Characteristics Using Different Definitions of Subsequent Response

\begin{tabular}{lccc}
\begin{tabular}{l} 
Subsequent response defined \\
as $\begin{array}{l}\text { a } \% \text { improvement in PANSS } \\
\text { total score }\end{array}$ \\
\cline { 2 - 4 }
\end{tabular} & $\mathbf{2 0 \%}$ & $\mathbf{3 0 \%}$ & $\mathbf{4 0 \%}$ \\
\hline Responder (n, \%) & $307(59.5)$ & $185(35.9)$ & $92(17.8)$ \\
Non-responder (n, \%) & $209(40.5)$ & $331(64.1)$ & $424(82.2)$ \\
Positive predictive value (PPV) (\%) & 86.2 & 68.8 & 42.0 \\
Negative predictive value (NPV) (\%) & 50.3 & 76.2 & 91.0 \\
Sensitivity (\%) & 38.8 & 51.4 & 63.0 \\
Specificity (\%) & 90.9 & 87.0 & 81.1 \\
Total accuracy (\%) & 59.9 & 74.2 & 77.9 \\
\hline
\end{tabular}

$n$, number in group; PANSS, Positive and Negative Syndrome Scale. Early responders to risperidone $(n=138)$; early non-responders to risperidone $(n=378)$. End point response status was defined by last available PANSS score between weeks 3 and I2; therefore, patients who dropped out between week 2 and week 3 were not counted in this analysis.

large a response with either continued exposure to risperidone or following a switch to olanzapine. Therefore, post hoc analyses were conducted for ENRs who met at least moderately ill criteria (58\%) at Week 2 and were repeated for those who did not (42\%). Among ENRs who were still at least moderately ill at Week 2, olanzapine-treated patients experienced significantly greater improvement in PANSS total score (LS mean change in PANSS total score from Week 2 to Week 12: ENR-RIS, -16.28; ENR-OLZ, -22.42; $p<0.05$ ), with separation between the ENR-OLZ and ENRRIS groups evident 6 weeks after randomization (LS mean change in PANSS total score at Week 8: ENR-RIS, -15.26; ENR-OLZ, $-19.04 ; p<0.05)$.

\section{Safety Parameters}

During Study Period II, no significant differences were observed in the frequency of serious adverse events between risperidone ER and ENR groups. A between-group comparison of treatment-emergent abnormal laboratory values observed in $\geqslant 2 \%$ of patients revealed a significant difference between ER and ENR groups for low urea nitrogen, as all 11 cases were observed in the ENR group $(p=0.041)$. No significant differences were observed in the frequency of extrapyramidal symptoms (ie, akathisia, dyskinesia, or parkinsonism) reported by ER $v s$ ENR groups.

During Study Period III, no serious adverse events were reported with significantly greater frequency between ER and ENR-RIS groups, or between ENR-RIS and ENR-OLZ groups (data not shown). Comparison of treatmentemergent adverse events (Table 3) revealed that lethargy was reported with significantly $(p=0.005)$ greater frequency in the ER group compared with the ENR-RIS group, whereas insomnia was reported with significantly $(p=0.038)$ greater frequency in the ENR-RIS group. Diarrhea was reported with significantly $(p=0.036)$ greater frequency in the ENR-RIS group compared with the ENROLZ group. No treatment-emergent adverse events were reported with significantly greater frequency among the ENR-OLZ group.

During Study Period III, the ENR-RIS group showed significantly greater increases in body mass index $(p=0.034)$ and body weight $(p=0.026)$ compared with the ER group. No significant difference in body mass index 
$(p=0.125)$ or weight change $(p=0.143)$ was observed between ENR-RIS and ENR-OLZ groups. The percentages of patients experiencing clinically significant weight gain (ie, $\geqslant 7 \%$ ) during Study Period III were: ERs, $n=5(3.6 \%)$, ENR-RIS, $n=24(12.8 \%)$, ENR-OLZ, $n=32$ (18.0\%); ER vs ENR-RIS, $p=0.005$; ENR-RIS $v s$ ENR-OLZ, $p=0.192$.

A significantly greater increase in triglycerides was observed for the ENR-OLZ group compared with the ENR-RIS group $(p=0.005)$. However, there were no significant between-group comparisons between ER and ENR-RIS groups or between ENR-RIS and ENR-OLZ groups for treatment-emergent categorical changes in triglycerides. There were no significant between-group

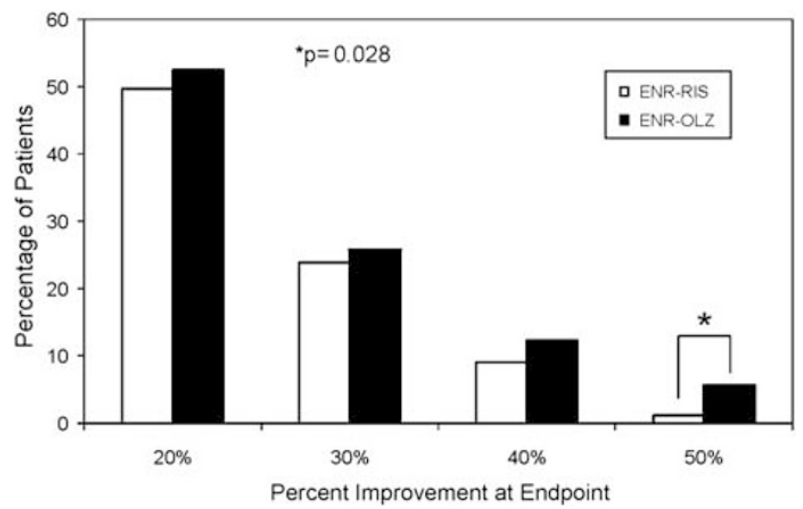

Figure 3 A comparison of the proportion of patients who met response criteria at end point between the two ENR groups. Responses are defined with varied cutoffs on percent change of PANSS total score from baseline. ENR-OLZ, early non-responder switched to olanzapine; ENR-RIS, early non-responder maintained on risperidone. differences between ENR-RIS and ENR-OLZ groups in mean or categorical change in glucose or cholesterol. There was a significant difference between ER and ENR-RIS groups for fasting glucose, with a shift from normal to diabetes (ER, $n=6$; ENR-RIS, $n=1 ; p=0.018$ ).

Both ENR groups showed a reduction in prolactin levels; however, the reduction in prolactin levels was significantly greater in the ENR-OLZ group compared with the ENR-RIS group $(p<0.001)$. No significant group differences were reported in the frequency of akathisia or parkinsonism, although treatment-emergent dyskinesia was reported with significantly greater frequency in the ENR-RIS group (9 patients, 5.2\%) compared with the ENR-OLZ group (1 patient, $0.6 \%)(p=0.020)$.

\section{DISCUSSION}

This study makes two major new observations. First, it prospectively shows that early response (at 2 weeks) is a strong and valid predictor of longer-term outcome. Second, it shows that this marker may be used to develop switch $v s$ stay algorithms that could be applied in clinical settings.

Compared with ENRs, ERs showed significantly greater improvement in overall psychopathology (PANSS total score) at all subsequent time points assessed up to 10 weeks, a finding consistent with our study hypothesis. In addition, compared with ENRs, ERs had higher proportions of responders (defined as $\geqslant 20,30,40$, and 50\% improvement in PANSS total score at end point), were more likely to attain normal to mild global illness severity, and achieved greater reduction in depressive symptoms. Early response,

Table 3 Treatment-Emergent Adverse Events Reported in $\geqslant 2 \%$ of All Patients During Study Period III

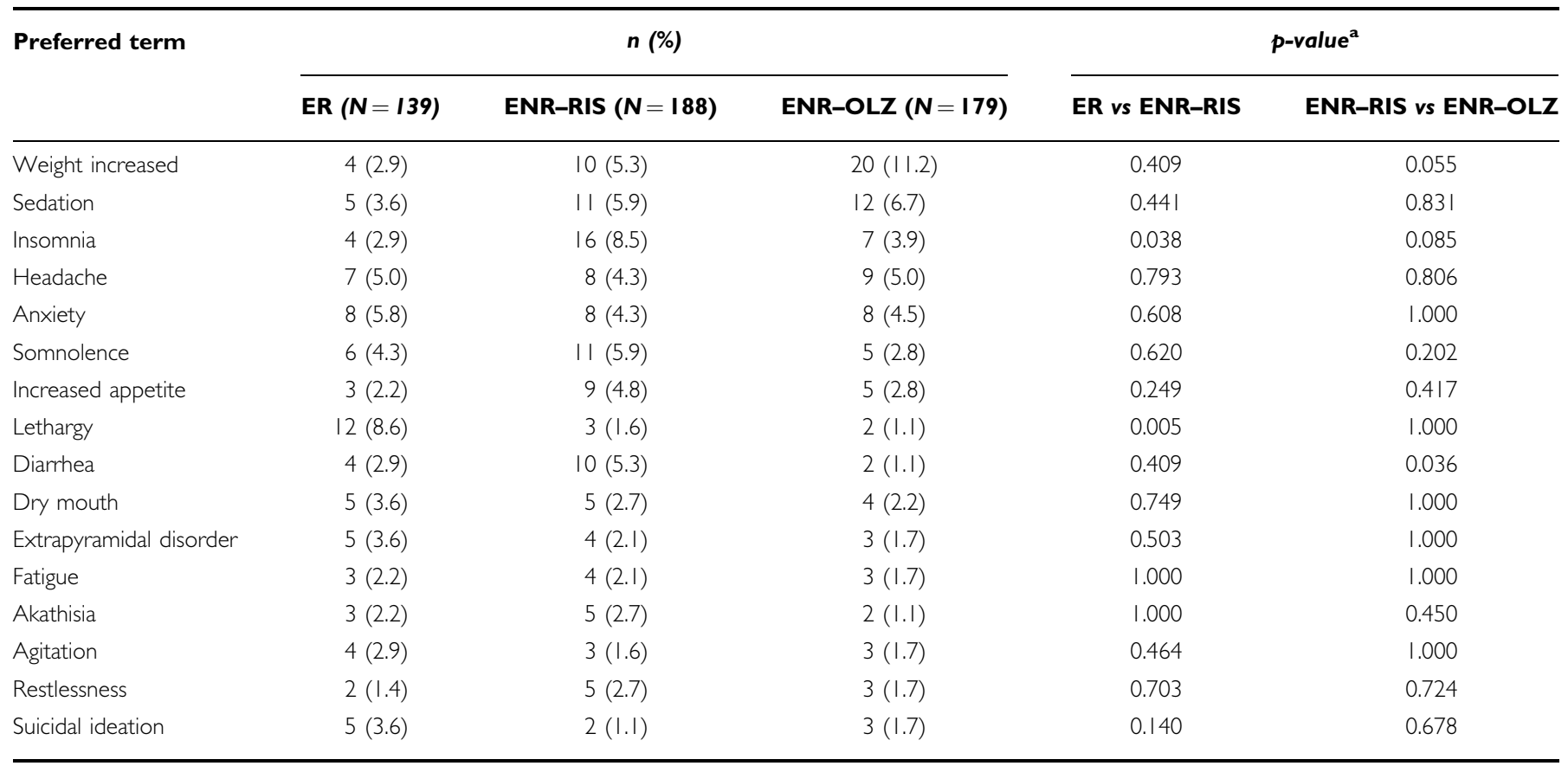

ENR, early non-responders; ER, early responders; $N$, number of patients; $n$, number of events; OLZ, olanzapine; RIS, risperidone.

${ }^{a} p$-value is from Fisher's exact test. 
defined as at least $20 \%$ improvement in PANSS total score at 2 weeks, was highly predictive of subsequent clinical outcomes during the following 10 weeks. Switching ENRs to olanzapine resulted in small but significantly greater improvement in overall psychopathology and in depressive symptoms compared with ENRs that were maintained on risperidone. The reduction in PANSS total score following the switch to olanzapine was greater among those patients who were still moderately ill at 2 weeks. Switching ENRs to olanzapine also resulted in significantly greater increases in triglycerides, a significantly greater decrease in prolactin, and significantly less treatment-emergent dyskinesia.

The rapid reduction in symptoms already evident by 2 weeks in patients identified as ERs is consistent with previous retrospective research showing that the onset of clinical response to an antipsychotic drug occurs early within the first week or two (Agid et al, 2003; Leucht et al, 2005a). Similarly, patients identified as ENRs were not likely to become responders with continued exposure as evidenced by relatively high NPV, a finding also supported by previous research (Ascher-Svanum et al, 2008; Correll et al, 2003; Kinon et al, 2008; Leucht et al, 2007, 2008). ENRs did not appear to be receiving a less than therapeutic level dose, as the ENR group received a significantly higher mean modal dose of risperidone during the first 2 weeks compared with ERs. Furthermore, there was no evidence at screening that patients who were subsequently identified as ERs or ENRs differed in regard to typical or atypical antipsychotic drug exposure or unmedicated status. Thus, early response/non-response status did not appear to reflect differences in medication status at the start of the study. Although all patients had to have experienced an exacerbation of their illness within the 2 weeks preceding visit 1, it was not possible to determine to what extent situational stress or cyclical variation in disease severity in patients who may be in a relatively stable condition may have led to an intensification in a patient's level of psychiatric care, nor what role such phenomena may have had in the observed early response/non-response dichotomization.

A sobering finding in this trial is that only a minority of patients who completed 2 weeks of risperidone therapy (28\%) were ERs, whereas approximately $72 \%$ of patients were ENRs. This finding is consistent with previous research that used data from patients treated with different atypical antipsychotics, including risperidone, showing that approximately $70 \%$ of patients were ENRs following 2 weeks of treatment (Kinon et al, 2008). A similarly high rate of early non-response was observed in patients treated with the first generation antipsychotic fluphenazine (Kinon et al, 1993), and in the naturalistic study of predominantly chronic schizophrenia outpatients treated with olanzapine, risperidone, or typical antipsychotics (Ascher-Svanum et al, 2008). The consistency of the current findings with previous research supports the modesty of benefits from an initial antipsychotic drug trial to most patients, suggesting the need to find alternative treatments. This is well supported by data from large trials showing that patients show a high rate of treatment discontinuation, and switching medications is common (Falkai, 2008; Haro et al, 2007; Lieberman et al, 2005).

Predictive parameters in our study revealed that although specificity was high and relatively consistent with previous work, the sensitivity for detecting subsequent response among ERs was higher than previously reported (AscherSvanum et al, 2008; Correll et al, 2003; Kinon et al, 2008). Furthermore, the total predictive accuracy was greatest using the $40 \%$ threshold for response criterion at the 12 week end point. This latter finding is consistent with our previous post hoc research in which the $40 \%$ threshold for response criterion observed at a 3-month end point was associated with greater total predictive accuracy (Kinon et al, 2008). Although the overall accuracy level is important, our findings of a high level of specificity and a high NPV appear to be of greater clinical utility given the need for clinicians to consider possible changes in the treatment regimen for ENRs. These findings highlight the important observation that proximal changes in symptom severity are indeed predictive of more distal patient outcomes.

In this study, we incorporated a switching component to assess if switching ENRs to olanzapine would facilitate overall symptom improvement compared with ENRs continuing on risperidone. Both the ENR-RIS and ENROLZ groups showed a steady decline in symptoms over the subsequent 10 weeks. Switching ENRs to olanzapine did result in significantly greater improvement at end point in overall psychopathology compared with ENRs who were maintained on risperidone therapy, although the difference in the PANSS total score observed between the two ENR groups was relatively small ( $\sim 3$-point difference) and statistically significant only after 10 weeks (12-week end point). The effect of switching on overall psychopathology was magnified further ( $\sim 6$-point difference) when the analysis focused on those ENRs who had remained moderately ill at 2 weeks, with separation between the ENR-OLZ and ENR-RIS groups evident after the initial 6 weeks of treatment. As a measure of clinical relevance, a 6-point differential in the PANSS total score has been used to test for noninferiority among antipsychotic drugs (Fleischhacker et al, 2009).

Apart from the dichotomization of patients into ER and ENR groups, there is likely a range of subsequent responses seen among ENRs. Some patients will continue to show little to no response with continued exposure to risperidone (ie, true non-responders) and these patients may be the ones who would benefit from a switch to another antipsychotic drug, whereas other patients may eventually reach the criterion of response with continued exposure to risperidone (ie, late responders), and still others would show little response even with a switch to another antipsychotic (ie, treatment resistant). In this study, although comparisons between the ENR groups across a range of categorical responses $(20,30$, and $40 \%$ ) had revealed no significant differences, a significant difference was observed for the percentage of patients achieving at least $50 \%$ reduction in PANSS total score at end point in the ENR-OLZ group compared with the ENR-RIS group. Although the significance of this finding is presently unknown, it may be suggestive that a subset of ENRs was highly responsive to olanzapine treatment. Although this study and others (Faries et al, 2008; Takahashi et al, 2006a) reported greater overall treatment outcomes following a switch from risperidone to olanzapine in those patients showing a limited response to risperidone, other studies (Ganguli et al, 
2008; Takahashi et al, 2006b) have reported improved outcomes following a switch from olanzapine to risperidone in patients showing a limited response to olanzapine. Thus, continued research is necessary to characterize these different patient subgroups and to identify the best treatment regimen, including the choice of an antipsychotic drug that will facilitate the greatest clinical outcome for each patient or subgroup of patients.

Collectively, these findings are consistent with previous studies showing a benefit of switching antipsychotic medications on symptom severity in patients with schizophrenia or schizoaffective disorder (Canive et al, 2006; Simpson et al, 2008; Takahashi et al, 2006a,b; Wang et al, 2006). Our data did not support the post hoc finding of 'no benefit' in switching antipsychotic medications in patients who were randomized to stay on their medication ('stayers') $v s$ those who were randomized to 'switched to another drug' ('switchers') reported from the CATIE trial (Essock et al, 2006; Rosenheck et al, 2009). Given that our study was prospective and included a control group of patients who were not switched, our findings would lend support to switching antipsychotic drugs as a reasonable approach to facilitate a patient's treatment response.

Switching ENRs to olanzapine also resulted in significantly greater increases in triglycerides, but significantly greater decreases in prolactin levels and significantly less treatment-emergent dyskinesia. No significant difference in body mass index or weight change was observed between ENR-RIS and ENR-OLZ groups. The greater increases in triglycerides and greater decreases in prolactin observed in olanzapine-treated patients compared with risperidonetreated patients are consistent with previous findings for triglycerides (Hardy et al, 2006; Kelly et al, 2008; Meyer et al, 2008); and prolactin (Kinon et al, 2006; Konarzewska et al, 2009), but not consistent with the previously observed changes in body mass index and/or weight (Kelly et al, 2008). Somewhat surprising, though, was the observed difference in the development of treatment-emergent dyskinesia reported in nine ENRs maintained on risperidone $(5.2 \%)$ compared with 1 ENR switched to olanzapine $(0.6 \%)$.

The finding that the effect of switching was greater when the analysis focused on ENRs who remained moderately ill at the 2-week end point may be important for refining future research on early prediction rules. Rather than focusing on a given percent reduction in symptoms alone, it may be necessary to combine the percent reduction in symptoms with a certain level of symptom severity (ie, moderately to severely ill) to adequately identify those patients who may benefit from a change in treatment.

\section{Limitations}

Despite its large sample size, prospective study design, and incorporation of a randomized, double-blind control arm, this study has several limitations.

First, this analysis focused only on early response/nonresponse in acutely exacerbated, chronically ill patients treated for schizophrenia. It is likely that the proportion of patients who will reach early responder status may differ for patients in different stages of the illness. Second, this study offers information only about switching early non-respon- ders from risperidone to olanzapine, leaving other switching options for future research.

Lastly, we used the full 30-item PANSS to assess early response and non-response, a lengthy measure that is not used in usual clinical practice, thus potentially limiting the clinical applicability of the finding in real-world settings. However, there may be a few symptom domains driving the early response/non-response distinction. Additional efforts are underway to determine whether a smaller set of PANSS items can result in similar predictive accuracy and, therefore, enable us to create a simple tool that clinicians could use to understand how their patients are responding early in treatment.

\section{Conclusions}

This is the first prospective, randomized, double-blind study to show that early response to an atypical antipsychotic (risperidone) is associated with greater subsequent symptom improvement and that early response/nonresponse reliably predicts subsequent clinical outcomes. Moreover, switching risperidone early non-responders to olanzapine following 2 weeks of treatment was found to facilitate further symptom improvement, especially among early non-responders who continued to be at least moderately ill. Some patients switching to olanzapine experienced changes in safety parameters, emphasizing the need to balance risks and benefits when determining appropriate treatment for an individual patient.

\section{ACKNOWLEDGEMENTS}

Appreciation is expressed to Heather Fox, Medical Information Specialist with Lilly USA, LLC, for administrative oversight of the editing for this article. We acknowledge Teri Tucker and Caron Modeas for editorial contributions. Ms Tucker and Modeas are medical editors employed fulltime by i3 Statprobe, a division of Ingenix, which is a subsidiary of United Health Group. Eli Lilly contracted the editing and formatting of this article with i3 Statprobe.

\section{DISCLOSURE}

Drs Kinon, Chen, Ascher-Svanum, Stauffer, and KollackWalker and Wei Zhou are all employees of Eli Lilly and Company. Dr John Kane serves as a Consultant and/or Advisory Board member for Bristol Meyer Squibb; Otsuka America Pharmaceutical Inc; Eli Lilly and Company; Janssen; Pfizer; Wyeth; Vanda Pharmaceuticals Inc.; GlaxoSmithKline; Lundbeck; Johnson \& Johnson; PGxHealth; and Proteus; is a shareholder of MedAvante; and serves on Speakers Bureaus for Bristol Meyer Squibb, Janssen, AstraZeneca, and Eli Lilly and Company. Dr Shitij Kapur has had affiliations with the following commercial organizations over the last 5 years: AstraZeneca; Bristol Meyers Squibb; Eli Lilly and Company; EMD Pharmaceuticals Inc.; Darmstadt; GlaxoSmithKline; Janssen; Neuromolecular Pharmaceuticals; Otsuka America Pharmaceutical Inc.; Organon Pharmaceuticals USA; Pfizer; Sanofi-Synthelabo; Servier; and Solvay Wyeth. Funding of this study was provided by Eli Lilly and Company. 


\section{REFERENCES}

Agid O, Kapur S, Arenovich T, Zipursky RB (2003). Delayed-onset hypothesis of antipsychotic action: a hypothesis tested and rejected. Arch Gen Psychiatry 60: 1228-1235.

Alvarez E, Ciudad A, Olivares JM, Bousoño M, Gómez JC (2006). A randomized, 1-year follow-up study of olanzapine and risperidone in the treatment of negative symptoms in outpatients with schizophrenia. J Clin Psychopharmacol 26: 238-249.

Ascher-Svanum H, Nyhuis AW, Faries DE, Kinon BJ, Baker RW, Shekhar A (2008). Clinical, functional, and economic ramifications of early nonresponse to antipsychotics in the naturalistic treatment of schizophrenia. Schizophr Bull 34: 1163-1171.

Barnes TR (1989). A rating scale for drug-induced akathisia. $\mathrm{Br} J$ Psychiatry 154: 672-676.

Canive JM, Miller GA, Irwin JG, Moses SN, Thoma RJ, Edgar JC et al (2006). Efficacy of olanzapine and risperidone in schizophrenia: a randomized double-blind crossover design. Psychopharmacol Bull 39: 105-116.

Conley RR, Mahmoud R (2001). A randomized double-blind study of risperidone and olanzapine in the treatment of schizophrenia or schizoaffective disorder. Am J Psychiatry 158: 765-774.

Correll CU, Malhotra AK, Kaushik S, McMeniman M, Kane JM (2003). Early prediction of antipsychotic response in schizophrenia. Am J Psychiatry 160: 2063-2065.

Davis JM, Chen N, Glick ID (2003). A meta-analysis of the efficacy of second-generation antipsychotics. Arch Gen Psychiatry 60: 553-564.

Dossenbach M, Erol A, el Mahfoud Kessaci M, Shaheen MO, Sunbol MM, Boland J et al (2004). Effectiveness of antipsychotic treatments for schizophrenia: interim 6-month analysis from a prospective observational study (IC-SOHO) comparing olanzapine, quetiapine, risperidone, and haloperidol. J Clin Psychiatry 65: 312-321.

Essock SM, Covell NH, Davis SM, Stroup TS, Rosenheck RA, Lieberman JA (2006). Effectiveness of switching antipsychotic medications. Am J Psychiatry 163: 2090-2095.

Falkai P (2008). Limitations of current therapies: why do patients switch therapies? Eur Neuropsychopharmacol 18: S135-S139.

Faries DE, Ascher-Svanum H, Nyhuis AW, Kinon BJ (2008). Switching from risperidone to olanzapine in a one-year, randomized, open-label effectiveness study of schizophrenia. Curr Med Res Opin 24: 1399-1405.

Fleischhacker WW, McQuade RD, Marcus RN, Archibald D, Swanink R, Carson WH (2009). A double-blind, randomized comparative study of aripiprazole and olanzapine in patients with schizophrenia. Biol Psychiatry 65: 510-517.

Ganguli R, Brar JS, Mahmoud R, Berry SA, Pandina GJ (2008). Assessment of strategies for switching patients from olanzapine to risperidone: a randomized, open-label, rater-blinded study. BMC Med 6: 17.

Guy W (1976). ECDEU Assessment Manual for Psychopharmacology Revised (Publication ADM 76-338) US Departmnet of Health, Education, and Welfare: Rockville, MD. pp 218-222.

Hardy TA, Marquez E, Kryzhanovskaya L, Taylor CC, Cavazzoni P (2006). Cross-sectional comparison of fasting lipids in normoglycemic patients with schizophrenia during chronic treatment with olanzapine, risperidone, or typical antipsychotics. J Clin Psychopharmacol 26: 405-408.

Haro JM, Suarez D, Novick D, Brown J, Usall J, Naber D et al (2007). Three-year antipsychotic effectiveness in the outpatient care of schizophrenia: observational versus randomized studies results. Eur Neuropsychopharmacol 17: 235-244.

Kay SR, Fiszbein A, Opler LA (1987). The positive and negative syndrome scale (PANSS) for schizophrenia. Schizophr Bull 13: 261-276.

Kelly DL, Conley RR, Love RC, Morrison JA, McMahon RP (2008). Metabolic risk with second-generation antipsychotic treatment: a double-blind randomized 8-week trial of risperidone and olanzapine. Ann Clin Psychiatry 20: 71-78.

Kinon BJ, Ahl J, Liu-Seifert H, Maguire GA (2006). Improvement in hyperprolactinemia and reproductive comorbidities in patients with schizophrenia switched from conventional antipsychotics or risperidone to olanzapine. Psychoneuroendocrinology 31: 577-588.

Kinon BJ, Chen L, Ascher-Svanum H, Stauffer VL, Kollack-Walker S, Sniadecki JL et al (2008). Predicting response to atypical antipsychotics based on early response in the treatment of schizophrenia. Schizophr Res 102: 230-240.

Kinon BJ, Kane JM, Johns C, Perovich R, Ismi M, Koreen A et al (1993). Treatment of neuroleptic-resistant schizophrenic relapse. Psychopharmacol Bull 29: 309-314.

Konarzewska B, Wolczynski S, Szulc A, Galinska B, Poplawska R, Waszkiewicz N (2009). Effect of risperidone and olanzapine on reproductive hormones, psychopathology and sexual functioning in male patients with schizophrenia. Psychoneuroendocrinology 34: 129-139.

Leucht S, Busch R, Hamann J, Kissling W, Kane JM (2005a). Early-onset hypothesis of antipsychotic drug action: a hypothesis tested, confirmed and extended. Biol Psychiatry 57: 1543-1549.

Leucht S, Busch R, Kissling W, Kane JM (2007). Early prediction of antipsychotic nonresponse among patients with schizophrenia. J Clin Psychiatry 68: 352-360.

Leucht S, Kane JM, Kissling W, Hamann J, Etschel E, Engel RR (2005b). What does the PANSS mean? Schizophr Res 79: 231-238.

Leucht S, Shamsi SA, Busch R, Kissling W, Kane JM (2008). Predicting antipsychotic drug response. Replication and extension to six weeks in an international olanzapine study. Schizophr Res 101: 312-319.

Lieberman JA, Stroup TS, McEvoy JP, Swartz MS, Rosenheck RA, Perkins DO et al (2005). Effectiveness of antipsychotic drugs in patients with chronic schizophrenia. $N$ Engl J Med 353: 1209-1223.

Meyer JM, Davis VG, McEvoy JP, Goff DC, Nasrallah HA, Davis SM et al (2008). Impact of antipsychotic treatment on nonfasting triglycerides in the CATIE Schizophrenia Trial phase 1. Schizophr Res 103: 104-109.

Montgomery SA, Asberg M (1979). A new depression scale designed to be sensitive to change. Br J Psychiatry 134: 382-389.

Overall JE, Gorham DR (1962). The brief psychiatric rating scale. Psychol Rep 10: 799-812.

Rosenheck RA, Davis S, Covell N, Essock S, Swartz M, Stroup S et al (2009). Does switching to a new antipsychotic improve outcomes? Data from the CATIE Trial. Schizophr Res 107: 22-29.

Simpson GM, Angus JW (1970). A rating scale for extrapyramidal side effects. Acta Psychiatr Scand Suppl 212: 11-19.

Simpson GM, O'Gorman CJ, Loebel A, Yang R (2008). Long-term improvement in efficacy and safety after switching to ziprasidone in stable outpatients with schizophrenia. CNS Spectr 13: 898-905.

Takahashi H, Kamata M, Yoshida K, Ishigooka J, Higuchi $\mathrm{H}$ (2006a). Switching to olanzapine after unsuccessful treatment with risperidone during the first episode of schizophrenia: an open-label trial. J Clin Psychiatry 67: 1577-1582.

Takahashi H, Yoshida K, Ishigooka J, Higuchi H (2006b). Switching to risperidone after unsuccessful treatment of olanzapine in the first-episode schizophrenia: an open trial. Prog Neuropsychopharmacol Biol Psychiatry 30: 1067-1072.

Tran PV, Hamilton SH, Kuntz AJ, Potvin JH, Andersen SW, Beasley Jr C et al (1997). Double-blind comparison of olanzapine versus risperidone in the treatment of schizophrenia and other psychotic disorders. J Clin Psychopharmacol 17: 407-418.

Wang X, Savage R, Borisov A, Rosenberg J, Woolwine B, Tucker M et al (2006). Efficacy of risperidone versus olanzapine in patients with schizophrenia previously on chronic conventional antipsychotic therapy: a switch study. J Psychiatr Res 40: 669-676. 